\title{
Determinação da capacidade antioxidante do extrato do pó da casca do abacaxi aplicando diferentes técnicas de extração
}

\author{
Determination of the antioxidant capacity of pineapple peel powder extract by applying different \\ extraction techniques \\ Determinación de la capacidad antioxidante del extracto de cáscara de piña en polvo aplicando \\ diferentes técnicas de extracción
}

Recebido: 22/07/2021 | Revisado: 30/07/2021 | Aceito: 02/08/2021 | Publicado: 07/08/2021

Marcelo Eduardo Alves Olinda de Souza ORCID: https://orcid.org/0000-0001-6447-4131 Instituto Federal de Educação, Ciência e Tecnologia do Sertão Pernambucano, Brasil

E-mail: marcelo.olinda@ifsertao-pe.edu.br

Milena da Rocha Gomes ORCID: https://orcid.org/0000-0001-9135-3706 Instituto Federal de Educação, Ciência e Tecnologia do Sertão Pernambucano, Brasil E-mail: miileenaagoomees@gmail.com

Vitória Maria Souza Candeias ORCID: https://orcid.org/0000-0002-3092-0997 Instituto Federal de Educação, Ciência e Tecnologia do Sertão Pernambucano, Brasil E-mail: vitória.candeias@hotmail.com

Nailton de Macedo Albuquerque Junior ORCID: https://orcid.org/0000-0001-6757-5114 Universidade Federal de Campina Grande, Brasil E-mail: junior.nailton99@gmail.com

Emilly Thayná Ferreira Januário ORCID: https://orcid.org/0000-0003-3055-7726 Instituto Federal de Educação, Ciência e Tecnologia do Sertão Pernambucano, Brasil E-mail: emilly_thayna@hotmail.com

Débora Andrade Lima ORCID: https://orcid.org/0000-0002-2338-5885 Instituto Federal de Educação, Ciência e Tecnologia do Sertão Pernambucano, Brasil E-mail: 1debora.andrade@gmail.com

Silvana Belém de Oliveira Vilar ORCID: https://orcid.org/0000-0001-8141-3373 Instituto Federal de Educação, Ciência e Tecnologia do Sertão Pernambucano, Brasil

E-mail: silvana.belem@ifsertao-pe.edu.br

\begin{abstract}
Resumo
O abacaxi é uma das frutas mais cultivadas e apreciadas no Brasil, de modo que junto com o aumento do seu processamento, vem o aumento de seus resíduos que na maioria dos casos são descartados, apesar de possuírem nutrientes relevantes. O objetivo do presente trabalho foi estudar o aproveitamento da casca do abacaxi do Submédio do Vale do São Francisco para a obtenção de óleo ou extratos com capacidade antioxidante por meio do emprego de técnicas de extração a baixa pressão como o sistema Soxhlet, maceração e extração assistida por ultrassom, utilizando solventes orgânicos de diferentes polaridades. Posteriormente foi verificado o rendimento dos extratos obtidos, a extração com etanol apresentou melhor resultado. Para o teor de fenólicos totais (TFT) destacou-se a técnica de maceração com 12723,52 mg/g, e para determinar a atividade antioxidante (AA), foram utilizados os radicais ABTS e DPPH, com resultados respectivos de 0,04084 e $0,03859 \mathrm{mMTrolx} / \mathrm{g}$ pelo método de maceração com etanol. Com isso pode-se concluir que a casca do abacaxi pode ser uma matéria-prima de significativo potencial na elaboração de extrato com características antioxidantes.
\end{abstract}

Palavras-chave: Aproveitamento; Frutas; Resíduo.

\begin{abstract}
The pineapple is one of the most cultivated and appreciated fruits in Brazil, so that along with the increase of its processing, comes the increase of its residues that in most cases are discarded, despite having relevant nutrients. The objective of the present work was to study the utilization of the pineapple peel from the Submédio do Vale do São Francisco to obtain oil or extracts with antioxidant capacity through the use of low pressure extraction techniques, such as the Soxhlet system, maceration and ultrasound-assisted extraction, using organic solvents of different polarities.
\end{abstract}


Afterwards, the yield of the extracts obtained was verified, the extraction with ethanol showed the best results. For the total phenolic content (TFT) the maceration technique stood out with $12723.52 \mathrm{mg} / \mathrm{g}$, and to determine the antioxidant activity (AA), the ABTS and DPPH radicals were used, with respective results of 0.04084 and $0.03859 \mathrm{mMTrolx} / \mathrm{g}$ by the ethanol maceration method. Thus, it can be concluded that the pineapple peel can be a raw material of significant potential in the preparation of an extract with antioxidant characteristics.

Keywords: Utilization; Fruit; Residue.

\section{Resumen}

La piña es una de las frutas más cultivadas y apreciadas en Brasil, por lo que junto con el aumento de su procesamiento, viene el aumento de sus residuos que en la mayoría de los casos son descartados, a pesar de tener nutrientes relevantes. El objetivo de este trabajo fue estudiar el uso de la cáscara de piña del Submédio do Vale do São Francisco para obtener aceite o extractos con capacidad antioxidante mediante el uso de técnicas de extracción a baja presión, como el sistema Soxhlet, la maceración y la extracción asistida por ultrasonidos, utilizando disolventes orgánicos de diferentes polaridades. Posteriormente, se verificó el rendimiento de los extractos obtenidos, siendo la extracción con etanol la que presentó el mejor resultado. Para el contenido fenólico total (TFT) destacó la técnica de maceración con 12723,52 $\mathrm{mg} / \mathrm{g}$, y para determinar la actividad antioxidante (AA) se utilizaron los radicales ABTS y DPPH, con resultados respectivos de 0,04084 y 0,03859 mMTrolx/g por el método de maceración con etanol. Por lo tanto, se puede concluir que la cáscara de la piña puede ser una materia prima de importante potencial en la preparación de extractos con características antioxidantes.

Palabras clave: Utilización; Fruta; Residuo.

\section{Introdução}

As atividades agroindustriais em todo o mundo são muito intensivas, por isso a cadeia produtiva produz uma quantidade expressiva de resíduos agroindustriais, que estão diretamente relacionados aos impactos ambientais. Esse fato tem levado a uma crescente busca por alternativas sustentáveis ao uso de substâncias orgânicas produzidas pela agroindústria (Silva, 2016).

O abacaxi é uma fruta tropical que contém alta concentração de açúcares, vitaminas e minerais e é produzida em larga escala no Brasil. O uso industrial da fruta é destinado à produção de suco e polpa, gerando uma grande quantidade de resíduos. Curiosamente, esse resíduo torna-se um subproduto, pois além da presença enzimática da bromelaína, também pode atuar como um composto antimicrobiano e antioxidante (Oliveira, 2018).

$\mathrm{O}$ crescimento das atividades agroindustriais tem influenciado na produção de elevadas quantidades de resíduos na agricultura, indústria e áreas urbanas (FAO, 2013; Pedrassolli et al., 2015; Silva, 2015). A indústria de alimentos, especialmente as que processam suco de frutas, acabam influenciando no descarte de uma grande quantidade de substratos que haviam sido negligenciados no meio ambiente, como bagaço, cascas e sementes (Nascimento Filho e Franco, 2015). A redução da quantidade de resíduos rejeitados na natureza, bem como o aproveitamento integral dos alimentos é uma opção para solucionar o impacto ambiental causado por esses resíduos gerados pela indústria de alimentos, permitindo assim o emprego das partes que seriam descartados, mas que contêm conteúdo nutricional expressivo (Silva et al., 2017).

Dessa forma, descobrir outro destino para os resíduos que não seja o descarte, tem sido foco de estudo para muitos pesquisadores, utilizando como alternativa principal a preparação de farinhas de frutas e vegetais e o emprego em produtos alimentícios de diversas áreas, principalmente na indústria de panificação, sempre visando o enriquecimento nutricional de formulações alimentícias comerciais. Essas formulações alternativas vêm sendo avaliadas quanto à sua qualidade nutricional e aceitação sensorial (Menon et al, 2014; Karovicová \& Magala, 2013; Ade et al, 2014).

As farinhas obtidas de partes não convencionais de alimentos, pode ser uma alternativa para elaboração de diversos subprodutos, como por exemplo extratos que possuem atividade bioativa presentes no material. Sendo assim, para a obtenção desses extratos muitas metodologias clássicas, designadas também de métodos convencionais para a extração de compostos bioativos, contudo esses métodos de extração apresentam desvantagens como alto consumo de energia, baixa eficiência e longo tempo de extração (Naffati et al., 2017). Assim, os extratos cuja composição possui compostos fenólicos antioxidantes, são de 
particular interesse para a indústria alimentícia, tendo com funcionalidade a conservação de alimentos, podendo ser usado como ingrediente funcional e/ou aditivo natural.

O estudo de alternativas para métodos de extração tem adquirido importante papel na obtenção de produtos e matériasprimas com súplica natural e para uso em formulações de alimentos, fármacos e cosméticos. Além disso, tem como objetivo a redução de tempo de processo, melhorias nos rendimentos e na qualidade final do produto (Coelho, 2015).

Sendo assim, devido a elevada preocupação quanto às questões ambientais em relação as partes não convencionais que tem como destino o descarte, o presente trabalho visa estudar o aproveitamento da casca do abacaxi para a obtenção de extratos, avaliando a eficiência das técnicas de extração (rendimento do processo) sobre a composição fenólica total e capacidade antioxidante.

\section{Metodologia}

O presente trabalho foi realizado no Instituto Federal de Educação, Ciência e Tecnologia do Sertão Pernambucano, Campus Petrolina-PE, no Laboratório Experimental de Alimentos (LEA) e Laboratório de Águas e Bebidas, seguindo a abordagem metodológica de forma quantitativa descrita por Pereira et al. (2018).

\subsection{Preparo da Matéria-prima}

As cascas do abacaxi (Ananas comosus L.) foram adquiridas em períodos de produção diferentes e no Submédio Vale do São Francisco e com diferentes estádios de maturação, em seguida sendo levados até o Laboratório Experimental de Alimentos (LEA), localizado no IF Sertão - PE /Campus Petrolina. Após a aquisição de frutas que apresentavam cascas isentas de danos físicos, no Laboratório Experimental de Alimentos (LEA), situado no IF Sertão - PE/Campus Petrolina foi realizado a separação das cascas da polpa, que em seguida foram sanitizadas a 100ppm/5min.

\subsection{Secagem}

As cascas do abacaxi (Ananas comosus L.) foram submetidos à secagem em secador por circulação de ar forçado (Desidratador Pardal PE 30, $220 \mathrm{~V}, 900 \mathrm{~W}$ ) na temperatura de $60^{\circ} \mathrm{C}$, sendo estes pesados a cada 1 hora até obtenção de peso constante.

Depois da secagem as cascas foram trituradas em liquidificador industrial (JL COLOMBO - 8 Litros Aço Inox) durante 5 minutos em velocidade média, até a obtenção de uma farinha, que foi peneirada em peneira granulométrica de aço inox (a bronzinox) de 10 mesh. Logo após, a farinha foi pesada, dividida em três porções, embaladas em papel alumínio seguidos de saquinhos plásticos de baixa densidade, e por fim foram selados e levados para armazenagem em freezer $\left(-18^{\circ} \mathrm{C}\right)$.

\subsection{Regentes}

Para a realização das extrações de baixa pressão foram usados os solventes etanol P.A. (EtOH), que é obtido através de fontes renováveis sendo comumente utilizado em indústrias alimentícias e não é toxico, e o hexano P.A. (Hx) que é o solvente orgânico mais usado no processo de extração de óleos, por possuir estreita faixa de ebulição, ser imiscível com a água e por ser mais seletivo.

Na Tabela 1, são apresentados os dados relacionados ao ponto de ebulição e polaridade destes solventes. 
Tabela 1. Ponto de ebulição e polaridade dos solventes.

\begin{tabular}{ccc}
\hline Solventes & Ponto de Ebulição $^{\mathbf{1}}$ & Polaridade $^{\mathbf{2}}$ \\
\hline Etanol & $78,37^{\circ} \mathrm{C}$ & 5,2 \\
\hline Hexano & $69{ }^{\circ} \mathrm{C}$ & 0 \\
\hline
\end{tabular}

\subsection{Extração a baixa pressão}

${ }^{1}$ Fonte: Carvalho (2016). ${ }^{2}$ Fonte: Souza (2015).

\subsubsection{Método Soxhlet}

As extrações para obtenção de extrato por sistema Soxhlet foram executadas de acordo com a metodologia do Instituto Adolfo Lutz (IAL - $4^{\mathrm{a}}$ edição, 2008). Foram pesadas 5 gramas das cascas do abacaxi (Ananas comosus L.), posteriormente postas em cartuchos de filtro de café e encaixados ao extrator de óleo e graxas (MARCONI, MA 491). No copo de extrator, foram depositados $150 \mathrm{~mL}$ de solvente, em seguida, acoplado no sistema, tendo o aquecimento ligado de acordo com o ponto de ebulição do solvente, tendo duração de 6 horas de extração.

\subsubsection{Método Maceração}

O método de maceração consiste em uma extração da matéria-prima a temperatura ambiente em recipiente fechado, com duração de 5 dias, sob agitação manual de 1 vez ao dia, utilizando solvente orgânico (Sachindra et al.,2006). Seguindo este método, em um frasco tipo âmbar, foram adicionadas 25 gramas da amostra e $100 \mathrm{~mL}$ de solvente para efetuar a extração. Passado o período estabelecido, foi realizada uma filtração a vácuo para remoção da amostra sólida do extrato.

\subsubsection{Método Ultrassom}

Foi utilizado o método adaptado de Gu et al. (2008) que consiste na extração da matéria-prima, a temperatura ambiente, adicionado $5 \mathrm{~g}$ de matéria-prima com $150 \mathrm{~mL}$ de solvente orgânico. Para isso, em balão volumétrico foi depositado a amostra e o solvente, onde este recipiente foi tampado e mergulhado em uma lavadora ultrassônica (USC-700/55 kHz, Unique Ind. e Com. de produtos Eletrônicos Ltda., Indaiatuba/SP, Brasil), durante o período de 60 min de extração. Ao final, foi feita uma filtração a vácuo para separação da amostra do extrato.

\subsubsection{Eliminação do solvente por rotaevaporação}

Ao final de cada extração, os extratos foram submetidos ao processo de remoção do solvente presente neles, em rotaevaporador (Fisatom 801 - Mod. 550 / 200 RPM; 230 V; 60 Hz; 1200 W, Brasil) com rotação de 60 rpm, em condições a vácuo e com temperatura próxima ou igual ao ponto de ebulição do solvente empregado. A amostra foi depositada em um balão de fundo chato sob rotação imerso no banho de água aquecida.

A rotação do frasco cria uma superfície de troca, devido ocorrer uma maior mistura, assim permitindo realizar a evaporação de maneira rápida (Souza, 2015). Após eliminação total do solvente, o extrato na sua forma pastosa foi pesado juntamente do frasco âmbar, qual havia sido pesado vazio com antecedência para realização do cálculo de rendimento. $\mathrm{O}$ acondicionamento destes recipientes contendo as amostras foi feito em freezer a $-18^{\circ} \mathrm{C}$.

\subsection{Determinação do rendimento global das extrações $\left(\mathbf{X}_{0}\right)$}

Silva (2004) define rendimento global de extrações como a quantidade total de composto oleico extraído de um elemento, sendo obtido através de solventes, em conjunto com as condições pré-estabelecidas. Dentre estas, pode-se citar o tempo de extração e a quantidade de matéria-prima utilizada. 
Foi calculado o rendimento das extrações $\left(\mathrm{X}_{\mathrm{o}}\right)$ por meio de uma equação que expressa à razão entre a massa de extrato ( $\mathrm{m}_{\mathrm{ext}}$ ) obtido e a massa do pó das cascas do abacaxi (Ananas comosus L.) ( $\mathrm{m}_{\mathrm{far}}$ ), demonstrada na equação 1.

Equação 1. Equação para cálculo de rendimento das extrações.

$$
\mathrm{Xo}(\%)=\frac{\mathrm{m}_{\mathrm{ext}}}{\mathrm{m}_{\mathrm{far}}} \times 100
$$

Onde:

$\mathrm{X}_{0}=$ rendimento global $(\%)$

$\mathrm{m}_{\text {ext }}=$ massa do extrato

$\mathrm{m}_{\text {far }}=$ massa pó das cascas do abacaxi.

\subsection{Caracterização dos compostos bioativos totais}

\subsubsection{Determinação do teor de fenólicos totais (TFT)}

Para a determinação do potencial fenólico foi utilizado o método espectrofotométrico com reagente Folin-Ciocalteu (Singleton e Rossi, 1965). As amostras reagiram em solução de carbonato de sódio 7,5\% e Folin-Ciocalteu 10\%, adaptado e validado por Natividade et al. (2013) sendo o fator de diluição igual a 10. Para os grupos hidroxi-fenólicos reduzirem o ácido fosfomolíbdico-fosfotúngstico, produzindo um complexo de coloração azul que absorve entre 620 e 740 nm com um comprimento de onda máximo em $725 \mathrm{~nm}$, aguardou-se 2 horas. Por fim após a leitura no espectrofotômetro, foi utilizada a equação da regressão calculada na curva de calibração com ácido gálico, considerando as diluições, e os resultados expressos em equivalentes ao ácido gálico conforme a equação 2 .

Equação 2. Curva de calibração com ácido gálico

$$
\gamma=1019,4 X-4,6166(\mathrm{mg} / \mathrm{g})
$$

Onde:

$\mathrm{X}=$ Absorbância

\subsubsection{Determinação da capacidade antioxidante}

Por conta da sua facilidade de execução e baixo custo, além de obtenção de resultados confiáveis, o potencial antioxidante obtido pelo método do DPPH (EAG e Trolox), segundo o método de Brand-Williams et al. (1995) é bastante usado. Assim, foram adicionados $100 \mu \mathrm{L}$ da amostra a $2,9 \mathrm{~mL}$ de solução DPPH, aguardou-se 30 min no escuro até a leitura. Em seguida foi obtida a equação de regressão de curva padrão elaborada com ácido gálico e trolox. (Equações 2 e 3).

Equação 3. Regressão ácido gálico

$$
\text { \%inibição }=\left[1-\frac{(\text { Abs Amostra })}{(\text { Abs Controle })}\right] \times 100
$$


Equação 4. Trolox (nMTrolx/l)

$$
\frac{(\% \text { inibição }-2,5215)}{58,847} \times F D
$$

Onde:

FD = Fator de diluição

A atividade antioxidante também foi determinada pelo método de ABTS (Trolox) ABTS (2,2'-azino-bis 3etilbenzeno-tiazolina-6-ácido sulfônico). O procedimento foi baseado em método desenvolvido por Miller et al. (1993) com adaptações feitas por Rufino et al. (2010). Sendo feito o cálculo utilizando a equação de regressão obtida de curva padrão elaborada com trolox.

\subsection{Análise Estatística}

Os resultados de rendimentos de extração, teor de fenólicos totais e da atividade antioxidantes obtidos nas amostras de casca do abacaxi, foram avaliados separadamente (conforme o tipo de extração e solvente utilizado) através da análise de variância (ANOVA) ao nível de 5\% de significância (p < 0,05), com o auxílio do software SPSS Statistics 17.0 aplicando o teste de Tukey que avalia as diferenças entre os pares de tratamentos entre si (Montgomery, 2005).

\section{Resultados e Discussões}

\subsection{Curva de secagem da amêndoa}

Após obter os dados experimentais a partir da secagem, verificou-se ao longo da secagem, as cascas do abacaxi demonstraram uma redução gradativa de peso, qual foi representada através de uma curva gráfica, onde a temperatura do ar de secagem utilizado no processo apresentou influência na curva de secagem, já que quanto maior a temperatura mais rápido se alcança a umidade de equilíbrio constante, que é atingida quando o material perde totalmente o seu teor de água, neste caso, sendo necessário um período de 12 horas para ele alcançar peso constante. Leva-se em consideração que a temperatura de $60{ }^{\circ} \mathrm{C}$ utilizada no trabalho é aplicada para produtos agrícolas devido ser intermediária e não agredir tanto o teor nutricional.

Com isso, a curva de secagem da amêndoa do caroço da manga no decorrer do tempo está representada na Figura 1 abaixo.

Figura 1. Curva de secagem da casca de abacaxi $\left(60^{\circ} \mathrm{C}\right)$.

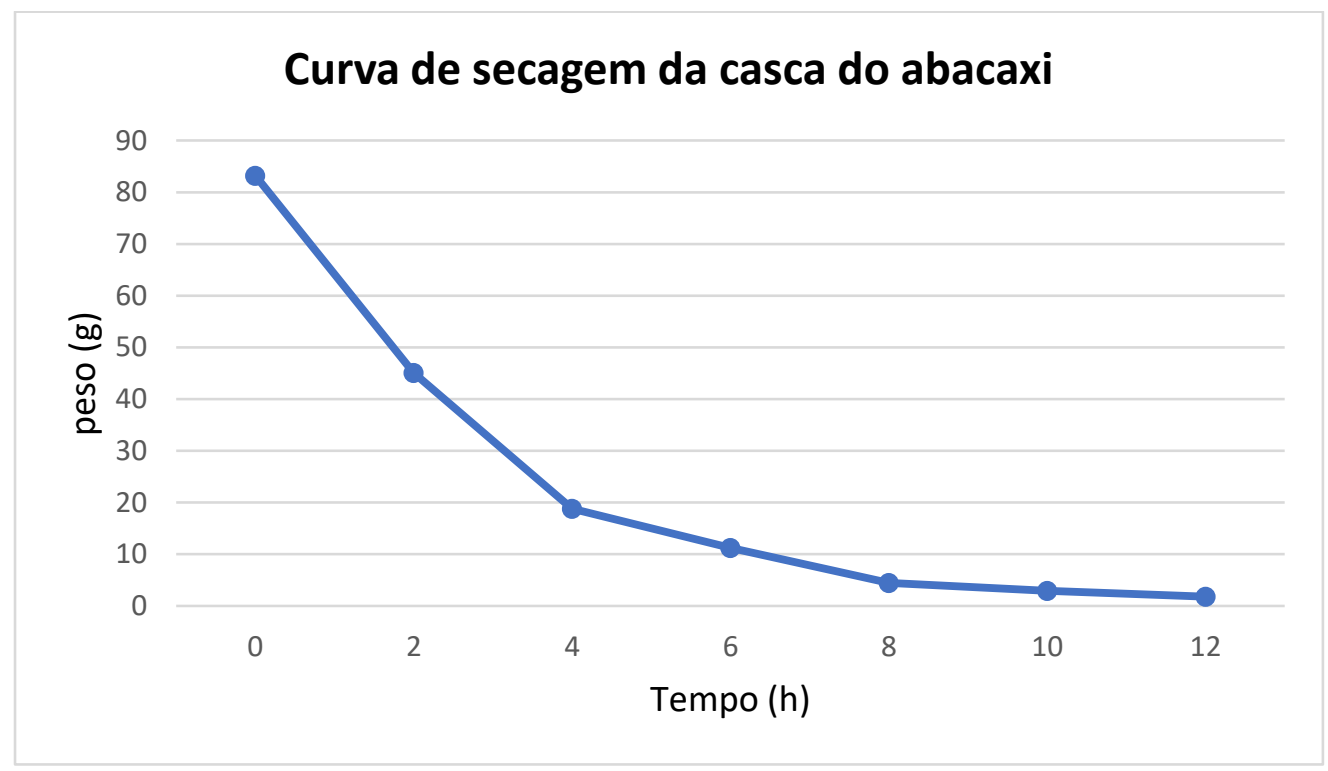

Fonte: Autores (2021). 
Dentro do secador houve uma troca de calor do ambiente quente com as cascas úmidas, ou seja, à medida que aumentou o tempo de exposição das cascas ao ar quente, maior foi a evaporação da água livre presente na matéria-prima. Assim pode-se observar a redução do teor de umidade da matéria-prima, até atingir um peso constante.

\subsection{Rendimento dos Extratos}

Com relação aos rendimentos de extratos obtidos, calculado conforme equação 1, foram alcançados os rendimentos de extração (em percentagens), que estão demonstrados na tabela 2, onde é possível visualizar o melhor desempenho de extração quanto aos métodos, em conjunto com os solventes.

Tabela 2. Rendimentos dos extratos.

\begin{tabular}{cccc}
\hline Solventes & Soxhlet & Ultrassom & Maceração \\
\hline Etanol & $41,63 \%$ & $16,14 \%$ & $12,68 \%$ \\
Hexano & $8,72 \%$ & $2,99 \%$ & $1,08 \%$
\end{tabular}

Fonte: Autores (2021).

Conforme os resultados de rendimento dos extratos (Tabela 2), foi observado que, entre os dois solventes e os métodos utilizados, o etanol aliado ao Soxhlet apresentou melhor desempenho.

O método de Soxhlet mostrou o maior rendimento em extração de óleo com relação aos demais, isso por que ele é realizado com aquecimento, além disso, o solvente fica em constante circulação sob o sistema

Em relação as extrações por maceração e assistida por ultrassom, quando comparadas ao do Soxhlet, foram obtidos menores rendimentos, provavelmente por serem métodos realizados em temperatura ambiente, o que fez com que a tensão superficial e viscosidade dos solventes permanecessem altas, o que possivelmente dificultou um maior contato com os compostos solúveis, e consequentemente reduzindo o percentual do extrato.

\subsection{Compostos Fenólicos Totais}

Os resultados de teor de fenólicos totais (método de Folin-Ciocalteu) obtidos no extrato do pó da casca de abacaxi, estão representados na Tabela 3.

Tabela 3. Fenólicos Totais do extrato do pó da casca de abacaxi.

\begin{tabular}{cccc}
\hline \multirow{2}{*}{ Solventes } & \multicolumn{3}{c}{ Métodos } \\
\cline { 2 - 4 } & Soxhlet & Ultrassom & Maceração \\
\cline { 2 - 4 } Etanol & $2468,35 \pm 992,61 \mathbf{a}$ & $2036,80 \pm 1218,78$ & $12723,52 \pm 859,28 \mathbf{a}$ \\
\hline Hexano & $1493,12 \pm 410,42 \mathbf{b}$ & - & $4731,42 \pm 562,92 \mathbf{b}$ \\
\hline
\end{tabular}

Fonte: Autores (2021).

De acordo com os resultados foi constatado que dentre as metodologias usadas, a que obteve melhor média foi a extração por meio da maceração juntamente com o etanol (Tabela 3), tendo estatisticamente diferença significativa $(\mathrm{p}<0,05)$. Além disso, o seu coeficiente de variação foi de 6,75\% que segundo Pimentel-Gomes (1985) na ausência de referências, valores menores que $10 \%$ indicam uma confiabilidade da média ótima, isso porque o coeficiente é baixo.

O extrato do pó da casca do abacaxi obtido pelo método de maceração aliada ao etanol também apresentou valores superiores de 12723,52 mg/g quando comparados a estudos de autores como Araújo et al., (2009) que obteve 24,89 mg/g em 
farinha de casca de manga. Ao avaliar os compostos fenólicos em resíduos de polpa de frutas, Sousa et al., (2011) relata que obteve $0,0911 \mathrm{mg} / \mathrm{g}$ em resíduo de polpa de abacaxi, $2,79 \mathrm{mg} / \mathrm{g}$ para acerola, 0,2411 mg/g em graviola, 0,0825 mg/g bacuri, e 0,0738 em cupuaçu (resíduo hidroalcóolico). Em farinha de resíduo de abacaxi de variedade não informada, Oliveira et al (2009) obteve o teor de fenólicos totais de 2,75 $\pm 38,0 \mathrm{mg} / \mathrm{g}$, em resíduo úmido de abacaxi (solvente metanol).

Sun et al. (2002), no estudo de atividade antioxidante de algumas frutas, encontraram o valor de $0,40 \mathrm{mg} / \mathrm{g}$ para polpa de abacaxi. Prado (2009) também encontrou valores semelhantes na polpa (em base úmida) do abacaxi de 0,41 mg/g. Todos eles também menores quando comparados ao atual estudo, possivelmente porque estudos declaram que as frutas são ricas em muitos nutrientes e compostos antioxidantes, mas que esses se concentram em grande parte nas cascas e sementes (MELO et al., 2008; Costa et al., 2000).

Não foi possível realizar nenhuma das três análises para a amostra submetida a extração por meio do processo de ultrassom com hexano, isso porque o seu rendimento não foi suficiente. O baixo rendimento foi possivelmente decorrente do que alguns autores como Liu et al. (2000) sugerem que o hexano por ser apolar não se classifica como um bom extrator.

\subsection{Capacidade Antioxidante}

A capacidade antioxidante pelos métodos de radicais livres ABTS e DPPH, obtidos no extrato do pó da casca de abacaxi, estão representados na Tabela 4 e 5 , respectivamente.

Tabela 4. Potencial de inibição de radicais livres pelo método DPPH (mMTrolx/g).

\begin{tabular}{cccc}
\hline \multirow{2}{*}{ Solventes } & & Métodos & Maceração \\
\cline { 2 - 4 } & Soxhlet & Ultrassom & $0,03859 \pm 0,01 \mathbf{a}$ \\
\hline Etanol & $0,1276 \pm 0,01 \mathbf{a}$ & $0,01273 \pm 0,00$ & $0,00852 \pm 0,00 \mathbf{b}$ \\
\hline
\end{tabular}

Fonte: Autores (2021).

Neste caso a extração com o solvente etanol por meio da maceração teve maior resultado de atividade antioxidante de 0,03859 (mMTrolx/g) conforme a tabela 4. Kuskoski et al., (2006) ao analisar a atividade antioxidantes em polpas de frutas encontrou o valor de $600 \pm 0,01$ TEAC (Capacidade Antioxidante Total Equivalente ao Trolox) $\mathrm{mM} / \mathrm{L}(0,6 \mathrm{mMTrolox} / \mathrm{g}$ ) para polpa de abacaxi (matéria fresca).

Além disso, o solvente que melhor apresentou desempenho foi o etanol em todas as extrações realizadas. O que confirma o que autores como Liu et al. (2000) que sugerem que solventes com alta polaridade, como a água, e com polaridade muito baixas, ou apolares, como o hexano, não são bons extratores.

Sousa et al. (2011) afirma que não existe um método oficial para determinação da atividade antioxidante em alimentos de origem vegetal e seus subprodutos, além da diversidade dos compostos bioativos podem ocorrer vários mecanismos antioxidantes. O que justifica as diferenças de resultados entre as amostras de cada autor.

Tabela 5. Potencial de inibição de radicais livres pelo método ABTS (mMTrolx/g).

\begin{tabular}{cccc}
\hline \multirow{2}{*}{ Solventes } & & Métodos & \\
\cline { 2 - 4 } & Soxhlet & Ultrassom & Maceração \\
\hline Etanol & $0,01298 \pm 0,00 \mathbf{a}$ & $0,02592 \pm 0,00$ & $0,04084 \pm 0,04 \mathbf{a}$ \\
\hline Hexano & $0,0037 \pm 0,01 \mathbf{b}$ & - & $0,01012 \pm 0,00 \mathbf{b}$ \\
\hline
\end{tabular}


Segundo a análise ABTS, a metodologia mais eficaz para extrair compostos antioxidantes foi a maceração com o solvente etanol, que apresentou $0,04084 \mathrm{mMTrolox} / \mathrm{g}$, conforme pode ser visualizando na tabela 5. Possivelmente por a maceração ser realizada a frio, ou seja, não utilizar fontes de calor pode ter influenciado nos resultados. O solvente etanol é considerado por muitos autores como sendo um bom extrator por sua natureza ser polar.

Os valores resultantes a partir do extrato aquoso do estudo de Vieira et al. (2011) para polpa de fruta congelada de bacuri e tamarindo respectivamente são de 0,094 e 0,075 (mMTrolox/g), são semelhantes ao resultado obtido da maceração e etanol desse estudo. Porém os extratos hidroalcóolicos também de Vieira et al. (2011) para as mesmas variedades anteriores respectivamente $(0,052$ e $0,114 \mathrm{mMTrolox} / \mathrm{g})$ são superiores ao desse estudo. Ao mesmo tempo vale ressaltar que anteriormente é entre frutas congeladas e casca do abacaxi

Leong e Shui (2002) classificou a goiaba com elevada capacidade, a laranja, mamão, manga e abacaxi com média capacidade e a melancia com baixa capacidade de sequestro através do radical ABTS (2,2'-azinobis-3-etilbenzotiazolina-6-ácido sulfônico). E ao realizarem análises de extratos provenientes de resíduos da polpa do abacaxi, Sousa et al. (2011) encontram valores de 0,110 \pm 0,017 / 0,090 0 0,002 TEAC (Capacidade Antioxidante Total Equivalente ao Trolox) (mMTrolox/g) para extrato aquoso e hidroalcóolico, respectivamente. Tais valores superiores ao encontrado nesse estudo, possivelmente por que a matéria-prima usada foi resíduo agroindustrial, que além de casca pode conter partes da polpa, também foi usado um solvente diferente, o álcool etílico 95\%.

Sousa et al. (2011) ainda analisou resíduos de outras frutas obtendo os resultados para extrato aquoso e hidroalcóolico respectivamente (mMtrolox/g) de goiaba $0,148 \pm 0,015$ e 0,421 $\pm 0,052$; acerola $0,518 \pm 0,103$ e 0,743 $\pm 0,127$; graviola 0,115 $\pm 0,023$ e $0,136 \pm 0,061$; bacuri $0,03667 \pm 0,005$ e $0,0735 \pm 0,009$; cupuaçu $0,177 \pm 0,048$ e $0,240 \pm 0,071$.

\section{Conclusão}

Diante dos resultados obtidos, foi observado que dentre todas as metodologias aplicadas para a extração dos compostos do pó das cascas do abacaxi (Ananas comosus L.), o solvente etanol juntamente com a extração por maceração apresentou maior eficiência para a extração de constituintes antioxidantes e compostos fenólicos das amostras.

O método usando ABTS se destacou quando comparado ao DPPH por apresentar uma maior capacidade de sequestro do radical. Assim, embora na literatura haja um vasto uso de diversos métodos, os valores do presente estudo mostraram-se bastante expressivos, indicando uma alto potencial fenólico e de moderada a baixa capacidade antioxidante da casca do abacaxi.

Com isso permite-se considerar que a casca do abacaxi é uma matéria-prima com potencial para elaboração de extrato com boa capacidade bioativa.

Aconselha-se a utilização de outras técnicas de extração, como também outros tipos de solvente, uma vez que as cascas do abacaxi (Ananas comosus L.) são partes não convencionais que apresentam boas características para elaboração de extratos com quantidades significativas de compostos bioativos totais.

\section{Agradecimentos}

Ao Instituto Federal do Sertão Pernambucano (IF-Sertão), pela concessão da bolsa de iniciação científica.

\section{Referências}

Ade. K. D., Lal, E. A., Rathid, A. S. (2014). Development and Quality Evaluation of Pineapple Pomace And Wheat Bran Fortified Biscuits. International Journal of Research in Engineering \& Advanced Technology, 2(3).

Araújo C.R., Melo, E.A., Lima G. V. L. A., Maciel, M. I. S. (2009). Resíduo agroindustrial de três variedades de mangas: teor de fitoquímicos e ação antioxidante. In: IX JEPEX - Jornada de ensino, pesquisa e extensão, Recife. Anais, UFRPE. p.42.

Brizola, R. \& Bampi, G. B. (2014). Desenvolvimento de barras alimentícias com adição de farinha de banana verde. Unoesc \& Ciência - ACBS, 5, 63-68. 
Coelho, R. A. (2015). Obtenção de óleo de sementes de quiuí (actinidia deliciosa) utilizando extração com solvente pressurizado e extração assistida com ultrassom. 75f. Tese (Doutorado em Engenharia de Alimentos), Universidade Federal do Paraná, Curitiba, PR.

Costa, R.P., Menendez, G., Bricarello, L.P., Elias, M.C., Ito, M. (2000). Óleo de peixe, fitosteróis, soja e antioxidantes: impactos nos lipídios e aterosclerose. Revista da Sociedade de Cardiologia, 10, 819-832.

Fao. Food and Agriculture Organization of the United States (2013). Produção brasileira de frutas 2013. Banco de dados. FAO.

Gu, Z., Chen, D., Han, Y., Chen, Z, Gu, F. (2008). Optimization of carotenoids extraction from Rhodobacter sphaeroides. LWT. 41, 1082-1088.

Instituto Adolfo Lutz (São Paulo). Métodos físico-químicos para análise de alimentos /coordenadores. (2008). Odair Zenebon, Neus Sadocco Pascuet e Paulo Tiglea - São Paulo: Instituto Adolfo Lutz, p.1020, versão eletrônica.

Karovicová, Z. J., Magala, M. (2013). Rheological and qualitative characteristics of pea flour incorporated cracker biscuits. Croat. J. Food Sci. Technol. 5, 1117.

Kim et al. (2002). Free radical scavenging activity of red ginseng aqueous extracts. Toxicology, 172, 149-156.

Kuskoski E. M.; Asuero, A. G; Maria Teresa Morales M, T;Fett, R. Frutos tropicais silvestres e polpas de frutas congeladas: atividade antioxidante, polifenóis e antocianinas. Cienc. Rural 36(4).

Lima, M. dos S., Silani, I. de S. V., Toaldo, I. M., Corrêa, L. C., Biasoto, A. C. T., Pereira, G. E., Bordignon-Luiz, M. T., Ninow, J. L. (2014). Phenolic compounds, organic acids and antioxidant activity of grape juices produced from new Brazilian varieties planted in the Northeast Region of Brazil. Food Chemistry, 161, 94-103.

Liu, F. F., Ang. C. Y. W., Springer, D. (2000). Optimization of extraction conditions for active components in Hypericum perforatum using surface methodology. Journal of Agriculture and Food Chemistry, 48, 3.364-3.371.

Macagnan, F. T., Moura, F. A., Santos, L. R., Bizzani, M., Silva, L. P. (2014). Caracterização nutricional e resposta sensorial de pães de mel com alto teor de fibra alimentar elaborados com farinhas de subprodutos do processamento de frutas. Boletim do Centro de Pesquisa de Processamento de Alimentos, 32.

Melo, E. A., Maciel, M. I. S., Lima, V. A. G. L., Nascimento, R. J. (2008). Capacidade antioxidante de frutas. Revista Brasileira de Ciências Farmacêuticas, 44, 193-201.

Menon, L., Majumdar, S. D., Ravi, U. (2014). Mango (Mangifera indicaL.) kernel flour as a potential ingredient in the development of composite flour bread. Indian Journal of Natural Products and Resources. 5, 75-82.

Naffati, A., Vladic J., Pavlic, B., Radosavljevic, R., Gavaric, A., Vidovic, S. (2017). Recycling of filter tea industry by-products: Application of subcriticalwater extraction for recovery of bioactive compounds from A. uvaursiherbal dust. Journal of Supercritical Fluids, 121, 1-9.

Nascimento Filho, W. B., Franco, C. R. (2015). Avaliação do potencial dos resíduos produzidos através do processamento agroindustrial no Brasil. Revista Virtual de Química, 7, 1968-1987.

Oliveira, A. A. N. (2018). Avaliação da Oxidação Lipídica em Hambúrguer de Carne Bovina Adicionado de Farinha da Casca do Abcaxi (Ananas comosus (L.) Merril) como Antioxidante Natural. Dissertação de Mestrado.

Oliveira, D. B., Pessanha, N. N. C., Bernardes, N. R., Silva, W. D., Muzitano, M. F., Oliveira, D. R (2009). Extrato dos frutos de Cereus Fernambucensis: Antioxidante e Inibição da Produção de Óxido Nítrico (NO) por Macrófagos. Inter Science place, 53-57.

Pedrassolli, I.M., Homem-Junior, A.C., Pandolfi, M.A.C. (2015). Aproveitamento dos resíduos de manga das agroindústrias. In: III SIMTEC - Simpósio de Tecnologia da FATEC. Anais. 3 n. 1. Taquaritinga.

Pereira, A. S., Shitsuka, D. M., Parreira, F. J., \& Shitsuka, R. (2018). Metodologia da Pesquisa Cientifica. UFSM.

Pimentel-Gomes, F. (1945). Curso de Estatística Experimental. (12a ed.), Livraria Nobel, 467p.

Prado, A. Composição fenólica e atividade antioxidante de frutas tropicais. (2009). 106 f. Dissertação (Mestrado em Ciência e Tecnologia de Alimentos) Universidade de São Paulo.

Re et al. (1999). Antioxidant activity applying and improved ABTS radical cation decolorization assay. Free Radical Biology Medicine, $26,1234-1237$.

Sachindra, N.M., Bhaskar, N., Mahendrakar, N.S. (2006). Recovery of carotenoids from shrimp waste in organic solvents. Waste Management, 26, 1092-1098.

Silva, C. S. (2016). Desenvolvimento de biscoitos enriquecidos com farinha de caroço de manga: incorporação de substâncias bioativas e aproveitamento de resíduos agroindustriais.2016. 59 f. Trabalho de Conclusão de Curso (Graduação). Universidade Federal Fluminense, Niterói.

Silva, D. C. de M. N. (2004). Determinação experimental de parâmetros de processos na extração supercrítica de óleo essencial de carquela (Baccaris trimera Less). Dissertação (Mestrado em Engenharia de Alimentos), Florianópolis: UFSC.

Silva, P. A. P., Costa Filho, D. V., Silva, A. J., Sousa, F. C. (2017). Aproveitamento de Resíduos Agroindustriais na Elaboração de Subprodutos. II Congresso Internacional das Ciências Agrárias.

Singleton, V. L., Rossi, J. A. (1965). Colorimetry of total phenolics with phosphomolybdic-phosphotungstic acid reagents. American Journal of Enology and Viticulture, 16, 144-158.

Sousa, M. S. B., Vieira, L. M., Lima, A. (2011). Fenólicos totais e capacidade antioxidante in vitro de resíduos de polpas de frutas tropicais. 14, $202-210$. 
Research, Society and Development, v. 10, n. 10, e155101018574, 2021

(CC BY 4.0) | ISSN 2525-3409 | DOI: http://dx.doi.org/10.33448/rsd-v10i10.18574

Souza, M. E. A. O. de et al. (2014). Aproveitamento de casca de manga Tommy Atkins (Mangifera indica Linn) para obtenção de extratos ricos em compostos fenólicos através de diferentes métodos de extração. XX Congresso Brasileiro de Engenharia Química, Florianópolis/SC.

Souza, M. E. A. O. de. (2015). Potencial antioxidante de extratos da casca de manga (Mangifera indica L.) da variedade Tommy Atkins obtidos por métodos a baixa e a alta pressão e dimensionamento de uma coluna para extração supercrítica. Florianópolis: UFSC. Tese (Doutorado em Engenharia de Alimentos), Departamento de Engenharia Química e Engenharia de Alimentos, Universidade Federal de Santa Catarina.

Sun, J.; Chu, Y.F.; Wu, X.; Liu, R.H. Atioxidant and antiproliferative activities of common fruits. Journal of Agricultural and Food Chemistry, 50, $7449-7454$.

Vieira, M. L., Sousa, B. S. M., Filho, M. J., Lima, A. (2011). Fenólicos Totais e capacidade antioxidante in vitro de polpas de frutos tropicais. 